Massimo Federico, Stefano Luminari, Alessandra Dondi, Luigi Marcheselli, and Stefano Sacchi, Clinica e di Sanità Pubblica, Università di Modena e Reggio Emilia, Modena; Alessandra Tucci, Azienda Ospedaliera "Spedali Civili," Brescia; Umberto Vitolo, Azienda Ospedaliera Universitaria "San Giovanni Battista," Torino; Luigi Rigacci, Azienda Ospedaliera Universitaria "Careggi," Firenze; Francesco Di Raimondo, Ospedale "Ferrarotto," Catania; Angelo Michele Carella, Ospedale "San Martino", Genova; Alessandro Pulsoni, Università "La Sapienza," Roma; Francesco Merli, Arcispedale "Santa Maria Nuova," Istituto di Ricovero e Cura a Carattere Scientifico (IRCCS), Reggio Emilia; Luca Arcaini, Fondazione IRCCS Policlinico "San Matteo," Università di Pavia, Pavia; Francesco Angrilli, Ospedale "Santo Spirito," Pescara; Caterina Stelitano, Ospedale "Bianchi, Melacrino, Morelli," Reggio Calabria; Gianluca Gaidano, Università del Piemonte Orientale Amedeo Avogadro, Novara; Matteo Dell'Olio, IRCCS Casa Sollievo della Sofferenza, San Giovanni Rotondo; Vito Franco, Università degli Stud di Palermo, Palermo; Sara Galimberti, Azienda Ospedaliera Universitaria Pisana, Pisa; and Maura Brugiatelli, Azienda Ospedaliera “Papardo," Messina, Italy.

Published online ahead of print at www.jco.org on March 25, 2013

Support information appears at the end of this article.

Authors' disclosures of potential conflicts of interest and author contributions are found at the end of this article.

Clinical trial information: NCT00774826.

Corresponding author: Massimo Federico, MD, Dipartimento di Oncologia ed Ematologia, Centro Oncologico Modenese,

Università di Modena e Reggio Emilia, Policlinico-Via del Pozzo 71, 41100 Modena, Italy; e-mail: federico@unimore.it.

C) 2013 by American Society of Clinical Oncology

0732-183X/13/3112-1506/\$20.00

DOI: 10.1200/JCO.2012.45.0866

\title{
R-CVP Versus R-CHOP Versus R-FM for the Initial Treatment of Patients With Advanced-Stage Follicular Lymphoma: Results of the FOLL05 Trial Conducted by the Fondazione Italiana Linfomi
}

Massimo Federico, Stefano Luminari, Alessandra Dondi, Alessandra Tucci, Umberto Vitolo, Luigi Rigacci, Francesco Di Raimondo, Angelo Michele Carella, Alessandro Pulsoni, Francesco Merli, Luca Arcaini, Francesco Angrilli, Caterina Stelitano, Gianluca Gaidano, Matteo Dell'Olio, Luigi Marcheselli, Vito Franco, Sara Galimberti, Stefano Sacchi, and Maura Brugiatelli

See accompanying editorial on page 1496

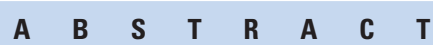

\section{Purpose}

Although rituximab $(R)$ is commonly used for patients with advanced follicular lymphoma (FL) requiring treatment, the optimal associated chemotherapy regimen has yet to be clarified.

\section{Patients and Methods}

We conducted an open-label, multicenter, randomized trial among adult patients with previously untreated stages II to IV FL to compare efficacy of eight doses of R associated with eight cycles of cyclophosphamide, vincristine, and prednisone (CVP) or six cycles of cyclophosphamide, doxorubicin, vincristine, and prednisone (CHOP) or six cycles of fludarabine and mitoxantrone (FM). The principal end point of the study was time to treatment failure (TTF).

\section{Results}

There were 534 patients enrolled onto the study. Overall response rates were 88\%, 93\%, and $91 \%$ for R-CVP, R-CHOP, and R-FM, respectively $(P=.247)$. After a median follow-up of 34 months, 3-year TTFs were 46\%, 62\%, and 59\% for the respective treatment groups (R-CHOP $\checkmark$ R-CVP, $P=.003$; R-FM $\vee$ R-CVP, $P=.006$; R-FM $v$ R-CHOP, $P=.763)$. Three-year progression-free survival (PFS) rates were 52\%, 68\%, and 63\% (overall $P=.011$ ), respectively, and 3 -year overall survival was $95 \%$ for the whole series. R-FM resulted in higher rates of grade 3 to 4 neutropenia (64\%) compared with R-CVP $(28 \%)$ and R-CHOP $(50 \% ; P<.001)$. Overall, 23 second malignancies were registered during follow-up: four in R-CVP, five in $\mathrm{R}-\mathrm{CHOP}$, and 14 in R-FM.

\section{Conclusion}

In this study, R-CHOP and R-FM were superior to R-CVP in terms of 3-year TTF and PFS. In addition, R-CHOP had a better risk-benefit ratio compared with R-FM.

\section{J Clin Oncol 31:1506-1513. (C) 2013 by American Society of Clinical Oncology}

\section{INTRODUCTION}

Follicular lymphoma (FL) is the most frequent indolent lymphoma subtype, accounting for $10 \%$ to $20 \%$ of all non-Hodgkin lymphomas in Western countries. At time of diagnosis, most patients have advanced disease, which requires systemic treatment. Although effective therapies are available, patients with FL still experience frequent relapses and shorter remissions at every disease recurrence. Recently, the outcome of FL has consistently improved, mainly as a result of the adoption of effective induction and salvage therapies. ${ }^{1}$
The current standard treatment for advanced FL includes immunochemotherapy (ICT), a combination of multiagent chemotherapy with anti-CD20 monoclonal antibody rituximab (R). Compared with chemotherapy alone, ICT has been associated with higher response, progression-free survival (PFS), and overall survival (OS) rates. ${ }^{2-5}$ Although the addition of $\mathrm{R}$ to chemotherapy is supported with the highest levels of evidence, less is known about which chemotherapy regimen should be chosen to achieve better results.

Several chemotherapy regimens have been adopted for the initial treatment of FL, ranging from 
single- to multiagent chemotherapies with or without anthracyclines. ${ }^{6,7}$ More recently, purine analogs have also been proposed. ${ }^{8-10}$ At present, the choice of initial therapy is still a matter of debate, with the anthracycline-containing regimen of cyclophosphamide, doxorubicin, vincristine, and prednisone ( $\mathrm{CHOP}$ ) being the most widely adopted so far. ${ }^{5,11,12}$

In 2006, the Fondazione Italiana Linfomi (formerly Intergruppo Italiano Linfomi) started a large prospective, randomized, multicenter trial to identify the best chemotherapy regimen to combine with $\mathrm{R}$ for first-line treatment of advanced FL. The study compared rituximab plus cyclophosphamide, vincristine, and prednisone (R-CVP) with $\mathrm{R}-\mathrm{CHOP}$ and rituximab plus fludarabine and mitoxantrone (R-FM) ICT regimens. Here we describe the final results of the study, which completed accrual in September 2010.

\section{PATIENTS AND METHODS}

The study was performed as a prospective, randomized, open-label, multicenter phase III trial. It was conducted in compliance with the Declaration of Helsinki, was approved by the appropriate research ethics committee, and required each patient to provide written informed consent before registration and random assignment.

The trial included previously untreated patients age 18 to 75 years, with histologically confirmed diagnosis of grade 1,2 , or 3a FL according to WHO classification, ${ }^{13}$ Ann Arbor stage II to IV disease, Eastern Cooperative Oncology Group performance status of 0 to 2, and active disease according to the Italian Society of Hematology guidelines. ${ }^{14}$ Patients ineligible for enrollment included those with grade 3b FL, Ann Arbor stage I disease, evidence of histologic transformation to diffuse large B-cell lymphoma, CNS involvement, or history of severe cardiac disease or previous malignancy other than in situ carcinoma of the cervix or basal cell carcinoma of the skin. Exclusion criteria also encompassed renal or hepatic function impairment not caused by lymphoma, known HIV infection, and hepatitis B (hepatitis B surface antigen positive) or C. Pregnant or lactating women and potentially childbearing women not using a reliable method of contraception were also excluded.

Central pathology review was performed for all patients with grade 3 FL or when grading was not specified in the local pathology report. For all other patients, no review was performed, and local diagnosis was accepted. Initial diagnostic and staging workup included physical examination; computed tomography (CT) scan with iodine contrast of the neck, chest, and abdomen; bone marrow (BM) biopsy; and complete set of laboratory tests. Positron emission tomography scan was not used for stage definition or response assessment.

\section{Random Assignment and Treatment Protocol}

Before registration, all patients signed an informed consent form. Eligible patients were randomly assigned according to a central procedure stratified by Follicular Lymphoma International Prognostic Index (FLIPI) score (0 to $2 v 3$ to 5$){ }^{15}$

The three study arms included eight doses of $\mathrm{R}$ combined with eight courses of CVP (control arm) or six cycles of CHOP or six cycles of FM, every 3 weeks. Drug doses and administration schedules for each regimen are summarized in Table 1. Growth factors were not routinely administered. Intermediate and final response assessments were required after cycle three and within 1 month after last R infusion, respectively. Patients showing progressive (PD) or stable disease (SD) were coded as experiencing treatment failure and shifted to salvage treatment at physicians' discretion. According to study protocol, maintenance therapy was not permitted.

Response assessment was to be performed with physical examination, laboratory tests, and total-body CT scan with contrast medium; BM biopsy was required only for assessment of final response if positive at baseline. During follow-up, disease status was to be assessed at months $+3,+6,+12$, $+18,+24$, and +36 with CT scan and with BM biopsy if positive at baseline.

\begin{tabular}{|c|c|c|c|}
\hline \multicolumn{4}{|c|}{$\begin{array}{l}\text { Table 1. Drug Doses and Time Schedules of the Three } \\
\text { Chemotherapy Regimens }\end{array}$} \\
\hline Drug & Dose $\left(\mathrm{mg} / \mathrm{m}^{2}\right)$ & Route & Days \\
\hline \multicolumn{4}{|l|}{$\overline{R-C V P *}$} \\
\hline Cyclophosphamide & 750 & IV & 1 \\
\hline Vincristine & $1.4 \dagger$ & IV & 1 \\
\hline Prednisone & 40 & $\mathrm{PO}$ & $1-5$ \\
\hline Rituximab & 375 & IV & 1 \\
\hline \multicolumn{4}{|l|}{$\mathrm{R}-\mathrm{CHOP} *$} \\
\hline Cyclophosphamide & 750 & IV & 1 \\
\hline Doxorubicin & 50 & IV & 1 \\
\hline Vincristine & $1.4 \dagger$ & IV & 1 \\
\hline Prednisone & 100 & $\mathrm{PO}$ & $1-5$ \\
\hline Rituximab & 375 & IV & 1 \\
\hline \multicolumn{4}{|l|}{ R-FM* ${ }^{*}$} \\
\hline Fludarabine & 25 & IV & $1-3$ \\
\hline Mitoxantrone & 10 & IV & 1 \\
\hline Rituximab & 375 & IV & 1 \\
\hline \multicolumn{4}{|c|}{$\begin{array}{l}\text { NOTE. Eight courses of R-CVP and six courses of R-CHOP and R-FM were } \\
\text { administered. For R-FM and R-CHOP, two additional courses (every } 21 \text { days) } \\
\text { of rituximab were scheduled. } \\
\text { Abbreviations: IV, intravenously; PO, orally; R-CHOP, rituximab plus cyclo- } \\
\text { phosphamide, doxorubicin, vincristine, and prednisone; R-CVP, rituximab plus } \\
\text { cyclophosphamide, vincristine, and prednisone; R-FM, rituximab plus fludara- } \\
\text { bine and mitoxantrone. } \\
\text { "Every } 21 \text { days. } \\
\text { tCapped at a total dose of } 2 \mathrm{mg} \text {. }\end{array}$} \\
\hline
\end{tabular}

\section{Statistics and Assessment of Efficacy}

Time to treatment failure (TTF) was the primary end point of the study, defined as time from date of study entry to last follow-up or to the first of the following events: less than partial remission (PR), shift to a different therapy for any reason after at least cycle one, PD or relapse, or death. Additional study end points were PFS, OS, response rate, and toxicity. PFS was measured from date of study entry to last follow-up or to one of the following events: PD, relapse, or death resulting from any cause. OS was defined from date of study entry to date of last observation or death resulting from any cause.

Response was assessed according to international criteria. ${ }^{16}$ Patients with negative baseline BM biopsy were classified as experiencing complete remission (CR) based on CT scan results, even if BM biopsy was not repeated at end of treatment. Toxicity was measured according to the standard Common Toxicity Criteria for Adverse Events (version 3.0; http://ctep.cancer.gov/ reporting/ctc.html) on a patient basis. The delivered dose-intensity was calculated according to Hryniuk, ${ }^{17}$ taking into account $\mathrm{R}$ as well.

The sample size was initially calculated at 252 patients according to the primary study end point (TTF) with the following initial assumptions: twosided 5\% significance test ( $\alpha$ error, 0.05 ) with a three-arm trial; power of $80 \%$ ( $\beta$ error, 0.2 ) with 3 years of uniform recruitment and 2 years of follow-up; and hazard ratio (HR) of 0.53 for TTF in the experimental arms, assuming median TTF of 32 months for R-CVP as reference. ${ }^{2}$ After enrollment of the first 214 patients, because actual accrual was faster than expected, the study was amended as follows: 4 years of uniform recruitment, 1 year of follow-up from last enrolled patient, and final accrual of 534 patients (178 patients per study arm). This allowed us to perform multiple comparisons among study arms, with a two-sided type I error controlled by the Bonferroni correction (0.05/ $3),{ }^{18}$ type II error of 0.10 ( $90 \%$ power), and $5 \%$ dropout rate. The initial hypothesis of an HR of 0.53 would be then realized with 200 events; with the hypothesis of an HR of 0.6, 211 events would have been required to achieve a test power of $84 \%$. An interim analysis of TTF was planned at $20 \%, 34 \%, 50 \%$, and $70 \%$ of the required events (approximately 200 events). To control the overall type I error, the Lan-DeMets alpha-spending function was used with O'Brien-Fleming boundaries of 0.0001, 0.0001, 0.0011, and 0.0042 at the interim analysis and 0.0115 at the final analysis for the two pairwise comparisons of R-CHOP versus R-CVP and R-FM versus R-CVP. 


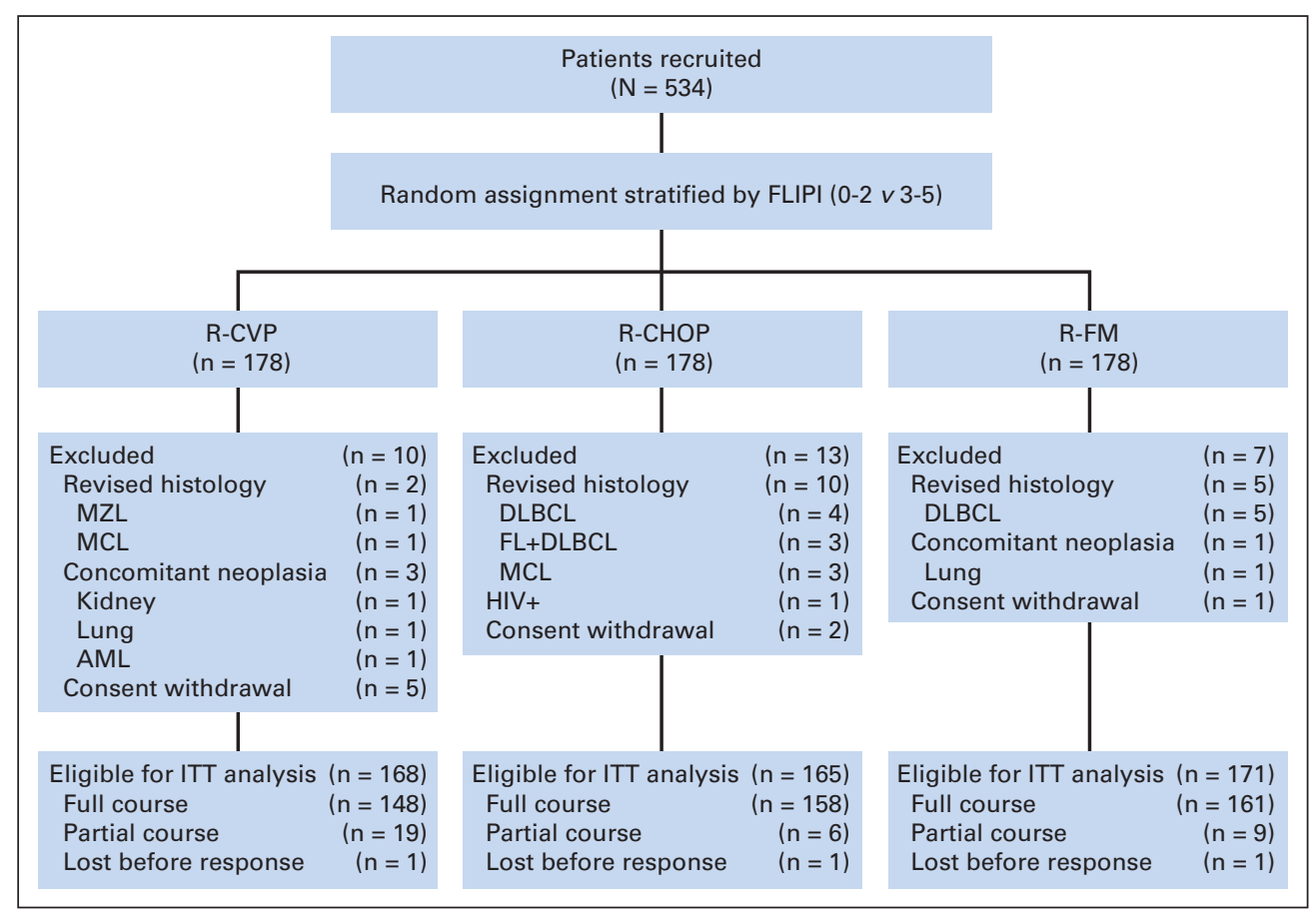

Fig 1. Treatment allocation and number of patients included in analysis, according to the CONSORT statement. ${ }^{19}$ After random assignment, 30 patients were considered ineligible and were subsequently excluded. $\mathrm{AML}$, acute myeloid leukemia; $\mathrm{DLBCL}$, diffuse large B-cell lymphoma; $F L$, follicular Iymphoma; FLIPI, Follicular Lymphoma International Prognostic Index; ITT, intention to treat; $M C L$, mantle cell lymphoma; $M Z L$, marginal zone lymphoma; $\mathrm{R}-\mathrm{CHOP}$, rituximab plus cyclophosphamide, doxorubicin, vincristine, and prednisone; R-CVP, rituximab plus cyclophosphamide, vincristine, and prednisone; R-FM, rituximab plus fludarabine and mitoxantrone.

Study end points were evaluated according to the intention-to-treat principle, with the provision that patients for whom exclusion criteria were discovered after random assignment or who withdrew their consent would be excluded from analysis. ${ }^{19}$ In the TTF analysis, the reported $P$ values in tables and figures were not adjusted for multiple comparisons. HRs for TTF and PFS were adjusted using FLIPI.

All statistical analyses were performed using STATA statistical software (release 10.1; College Station, TX). Survival curves were calculated using Kaplan-Meier estimates, ${ }^{20}$ and statistical comparisons between curves were made using the log-rank test. Post hoc comparisons between TTF and PFS, adjusted by potentially confounding factors, were performed using the Cox proportional hazards regression method. ${ }^{21}$ Proportionality of hazard was checked graphically by means of scaled Schoenfeld residuals. ${ }^{22}$ The $\chi^{2}$, Fisher's exact, and Kruskal-Wallis tests were used to compare variables when appropriate. ${ }^{23}$ All statistical comparisons were two sided.

\section{RESULTS}

Between March 2006 and September 2010, 534 patients were enrolled onto the FOLL0 5 trial by 58 Italian institutions, with 178 patients in each study arm. Thirty patients (6\%) were subsequently excluded (unconfirmed histology, 17; consent withdrawal, eight; synchronous cancer, four; HIV positivity, one), and the remaining 504 patients were analyzed (Fig 1). As shown in Table 2, patients were homogeneously distributed.

Planned interim analyses performed during the enrollment period allowed continuation of the trial. Median overall delivered doseintensities were $0.956,0.964$, and 0.918 for R-CVP, R-CHOP, and R-FM, respectively. Response was available for 501 patients.

At the end of induction therapy, the three arms (R-CVP, R-CHOP, and R-FM) had similar CR rates: $67 \%$ (95\% CI, 60\% to $74 \%$ ), $73 \%$ (95\% CI, $65 \%$ to $78 \%$ ), and $72 \%$ (95\% CI, $65 \%$ to $78 \%$ ), respectively ( $P=.543)$; overall response rates (ORRs; CR plus PR) were $88 \%$ (95\% CI, $82 \%$ to $93 \%$ ), $93 \%$ (95\% CI, $88 \%$ to $97 \%$ ), and
91\% (95\% CI, $86 \%$ to $95 \%)$, respectively ( $P=.247)$. After median follow-up of 34 months (range, 1 to 70 months), 213 failures were recorded, as summarized in Table 3. Overall 3-year TTF was 56\%.

In terms of TTF, R-CHOP and R-FM were significantly better than R-CVP $(62 \%$ and $59 \% v 46 \%$ at 3 -years; $P=.003$ and .006 , respectively; Fig 2A). No differences were observed between $\mathrm{R}-\mathrm{CHOP}$ and R-FM $(P=.763)$. HRs between R-CHOP versus $\mathrm{R}-\mathrm{CVP}$ and R-FM versus R-CVP, adjusted by FLIPI ( 0 to 2 v 3-5), were 0.62 (95\% CI, 0.44 to 0.86 ) and 0.63 (95\% CI, 0.46 to 0.87$)$, respectively.

Overall, 184 patients developed PD, including 13 PD during therapy, 11 PD from SD, 152 relapses or progressions from CR or PR, and eight deaths in CR or PR, thus resulting in a 3-year PFS of $61 \%$. Better PFS rates were observed for R-CHOP and R-FM over R-CVP (68\% and $63 \% v 52 \%$ at 3 years), with adjusted HRs for R-CHOP versus R-CVP and R-FM versus R-CVP of 0.64 (95\% CI, 0.45 to 0.92 ) and 0.66 (95\% CI, 0.47 to 0.94$)$, respectively (Fig 2B).

At final follow-up, 31 deaths were recorded. Because the study was not powered for OS analysis, no comparison among study arms was performed. Lymphoma-related deaths included PD or recurrence $(n=19)$; other causes of death were secondary malignancy $(n=5)$, heart failure $(\mathrm{n}=3)$, infection $(\mathrm{n}=2)$, cachexia nonlymphoma related $(\mathrm{n}=1)$, and car accident $(\mathrm{n}=1)$. OS at 3 years was $95 \%(95 \%$ CI, $92 \%$ to $97 \%$ ).

Study regimens were generally well tolerated. Patients treated with R-FM had the highest rate of grade 3 to 4 neutropenia, followed by those receiving R-CHOP and R-CVP (64\% $v$ 50\% and 28\%). Frequency of grade 3 to 4 thrombocytopenia was higher with R-CHOP and R-FM ( $3 \%$ and $8 \%)$ than with R-CVP $(0 \% ; P<.001)$. Overall, grade 3 to 4 infections occurred in 17 patients at a rate of $2.4 \%$, $3 \%$, and $4.7 \%$ for R-CVP, R-CHOP and R-FM, respectively $(P=.527$; Appendix Table A1, online only). Granulocyte colony-stimulating factor was used at least once during therapy in $46 \%$ of patients: $30 \%$ in 


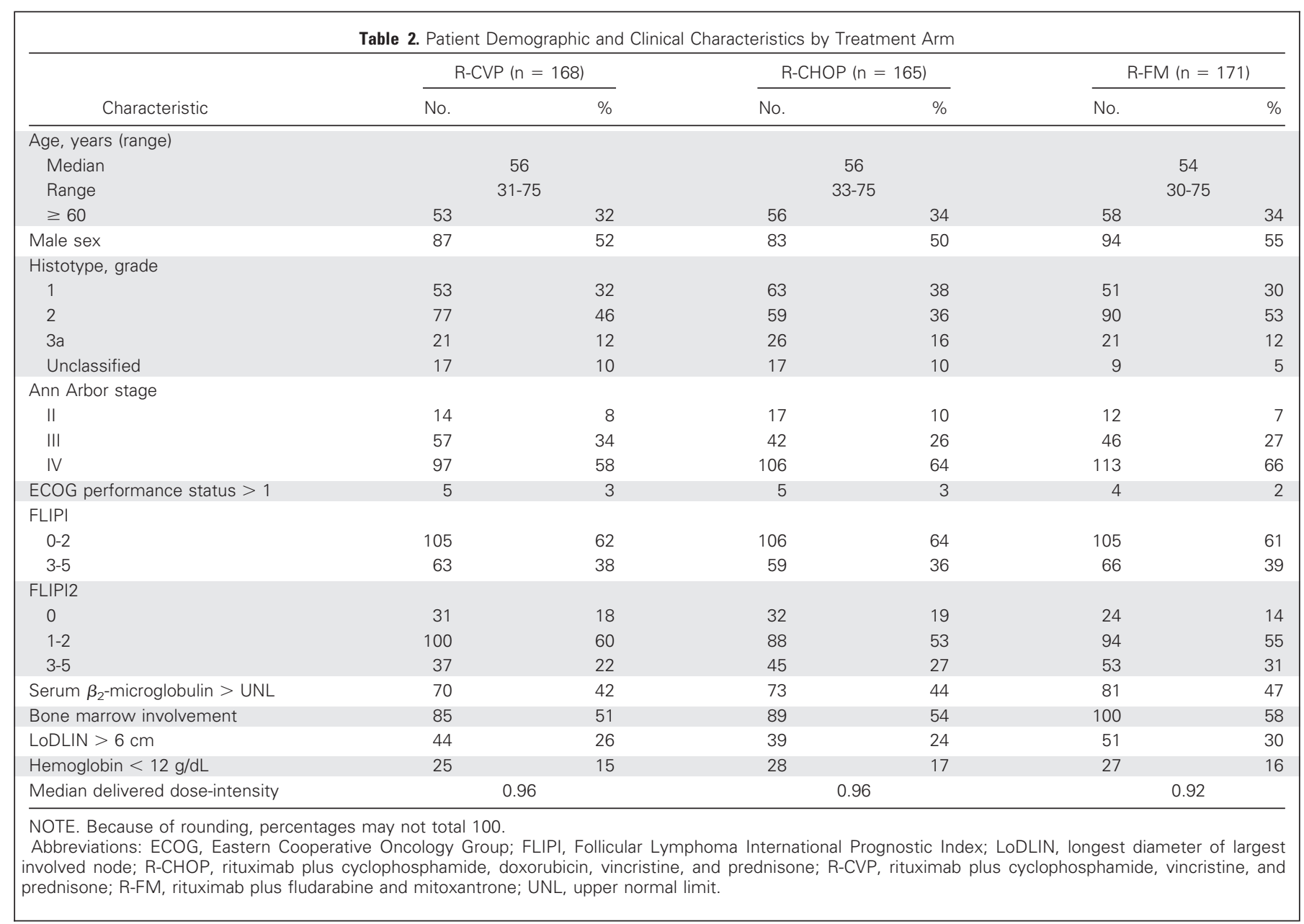

R-CVP, 52\% in R-CHOP, and 57\% in R-FM arms, respectively $(P<$ $.001)$. Nonhematologic toxicity was similar in all three arms, with a marginal excess of neurotoxicity in R-CVP; cardiac events were reported in five patients and were graded 3 to 4 in two (one each for $\mathrm{R}-\mathrm{CVP}$ and R-CHOP). There were no treatment-related deaths.

During follow-up, 23 patients were diagnosed with a second malignancy: four in R-CVP (one colon cancer, one prostate cancer, one Kaposi's sarcoma, and one breast cancer), five in R-CHOP (three lung cancers, one glioblastoma, and one endometrial cancer), and 14 in R-FM (three acute myeloid leukemias, one chronic myelomonocytic leukemia, two squamous cell skin cancers, one cervical cancer, one uterine cancer, one colon cancer, one prostate cancer, one lung cancer, one Warthin's cancer, one breast cancer, and one Hodgkin lymphoma).

We performed a post hoc exploratory analysis of patient subgroups categorized by age, hemoglobin level, size of largest involved node, $\beta_{2}$-microglobulin, BM involvement, and FLIPI (Figs 3A and

\begin{tabular}{|c|c|c|c|c|c|c|c|c|}
\hline Status & No. & $\%$ & No. & $\%$ & No. & $\%$ & No. & $\%$ \\
\hline$<P R$ & 19 & 11 & 10 & 6 & 14 & 8 & 43 & 8 \\
\hline Therapy shift & 9 & 5 & 4 & 2 & 7 & 4 & 20 & 4 \\
\hline Maintenance & 3 & 2 & 5 & 3 & 2 & 1 & 10 & 2 \\
\hline Total & 168 & & 165 & & 171 & & 504 & \\
\hline
\end{tabular}

NOTE. Because of rounding, percentages may not total 100

Abbreviation: CR, complete remission; PR, partial remission; R-CHOP, rituximab plus cyclophosphamide, doxorubicin, vincristine, and prednisone; R-CVP, rituximab plus cyclophosphamide, vincristine, and prednisone; R-FM, rituximab plus fludarabine and mitoxantrone; TTF, time to treatment failure. 


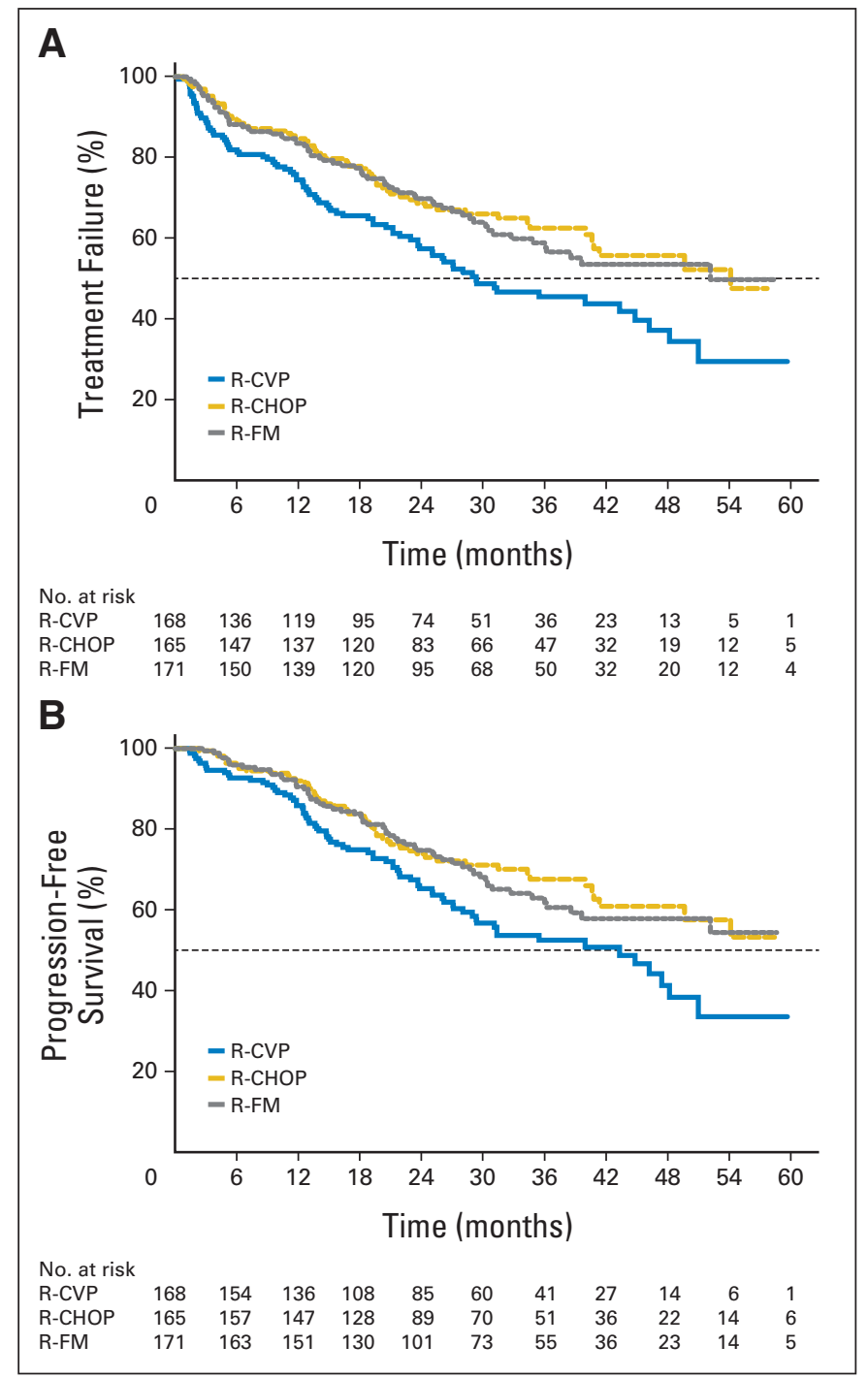

Fig 2. Kaplan-Meier analysis of probability of $(A)$ time to treatment failure and (B) progression-free survival according to intention-to-treat principle. R-CHOP, rituximab plus cyclophosphamide, doxorubicin, vincristine, and prednisone; R-CVP, rituximab plus cyclophosphamide, vincristine, and prednisone; R-FM, rituximab plus fludarabine and mitoxantrone.

3B). In addition, an unplanned exploratory analysis to assess potential relationship between FLIPI2 and treatment suggested the highest benefit with R-CHOP and R-FM over R-CVP in the larger group of patients at intermediate risk (FLIPI2, 1 to 2; data not shown).

\section{DISCUSSION}

The results of the FOLL05 randomized study clearly demonstrated that both R-CHOP and R-FM chemotherapy regimens lead to significantly improved TTF and PFS compared with R-CVP in patients with previously untreated FL. However, R-FM was associated with higher incidence of hematologic toxicity and late events compared with R-CHOP and R-CVP. Moreover, the 3-year OS rate of 95\% (95\% CI, $92 \%$ to $97 \%$ ) reinforces the assumption that end points such as TTF and PFS are more realistic than OS for clinical trials involving patients with $\mathrm{FL}^{24}$
When the study started in 2006, data from randomized trials confirmed that $\mathrm{R}$ added to any kind of chemotherapy improved treatment results. ${ }^{3-5,25}$ The FOLL05 study was designed to compare the three most commonly adopted regimens to define which one should be used as the treatment of choice: R-CHOP, R-CVP, and R-FM. Before the FOLL05 trial, almost all studies investigating the role of anthracyclines in FL were based on retrospective series and/or included patients with FL and other low-grade lymphomas. ${ }^{6,26,27}$ Moreover, results obtained with chemotherapy in the pre-R era needed to be reassessed under the conditions of highly effective ICT combinations. The main findings of the FOLL05 study were that chemotherapy choice still affects treatment results in the R era and that the addition of doxorubicin to CVP is required to improve efficacy of initial therapy. As shown in the post hoc analysis of TTF and PFS, the benefit of anthracycline was evident in almost all subgroups, except in those at high risk according to FLIPI. However, this latter finding that seemingly contrasts with current expectations and with recent data ${ }^{28}$ may have resulted from the small number of patients in the high-risk group, which did not translate the risk ratios of 0.63 and 0.79 observed for TTF and PFS, respectively, into statistically significance. Interestingly, compared with R-CVP, R-CHOP was not associated with higher risk of cardiac toxicity, which was reported at a low rate across the study.

Our results were compared with similar studies: the R-CVP arm of the randomized trial by Marcus et $\mathrm{al}^{25}$ and the R-CHOP arm of the German Low-Grade Lymphoma Study Group (GLGSG) randomized study. ${ }^{3}$ All studies included similar populations of adult patients with advanced FL. R-CVP treatment modalities were also the same, and both ORR and PFS were superimposable, suggesting that the R-CVP arm used in our study has highly reproducible results and worked at its best. R-CHOP ORR was similar to that published in the GLGSG study, whereas PFS was lower in our trial because of the adoption of both postinduction therapies with interferon and high-dose therapies in responding patients in the GLGSG trial.

When the study was designed, fludarabine was quoted in the literature as an effective drug for FL treatment, either alone or in combination. ${ }^{29,30}$ Additionally, FM was compared with CHOP in a phase III trial and was proposed as a more effective chemotherapy for first-line treatment of FL in terms of both clinical and molecular responses. ${ }^{30}$ On the basis of these results, it seemed to us appropriate to assess the efficacy of a fludarabine-containing regimen with concomitant R. The FOLL05 study concludes that both CHOP and FM with concomitant $\mathrm{R}$ have the same antilymphoma profile and are both superior to R-CVP in terms of TTF and PFS. These results compare favorably with those achieved in other phase II trials investigating $\mathrm{R}$ with fludarabine-based regimens. ${ }^{8-10,31}$ The FOLL05 study, however, suggests that fludarabine-based regimens have a lower therapeutic index than R-CHOP because of the higher frequency of adverse events, mainly hematologic, and, most importantly, the higher observed number of second malignancies. Among the 171 patients randomly assigned to receive R-FM, 14 developed a second malignancy, suggesting a higher risk for second cancer associated with fludarabinecontaining regimens, as already described in some retrospective reports on indolent lymphoma. ${ }^{32}$

At time of current analysis, with median follow-up of 34 months, the observed 95\% OS rate confirms the excellent results achieved with ICT in patients with FL. ${ }^{3,4,25,33}$ No further analysis was performed, and additional data are provided on OS because the limited statistical 


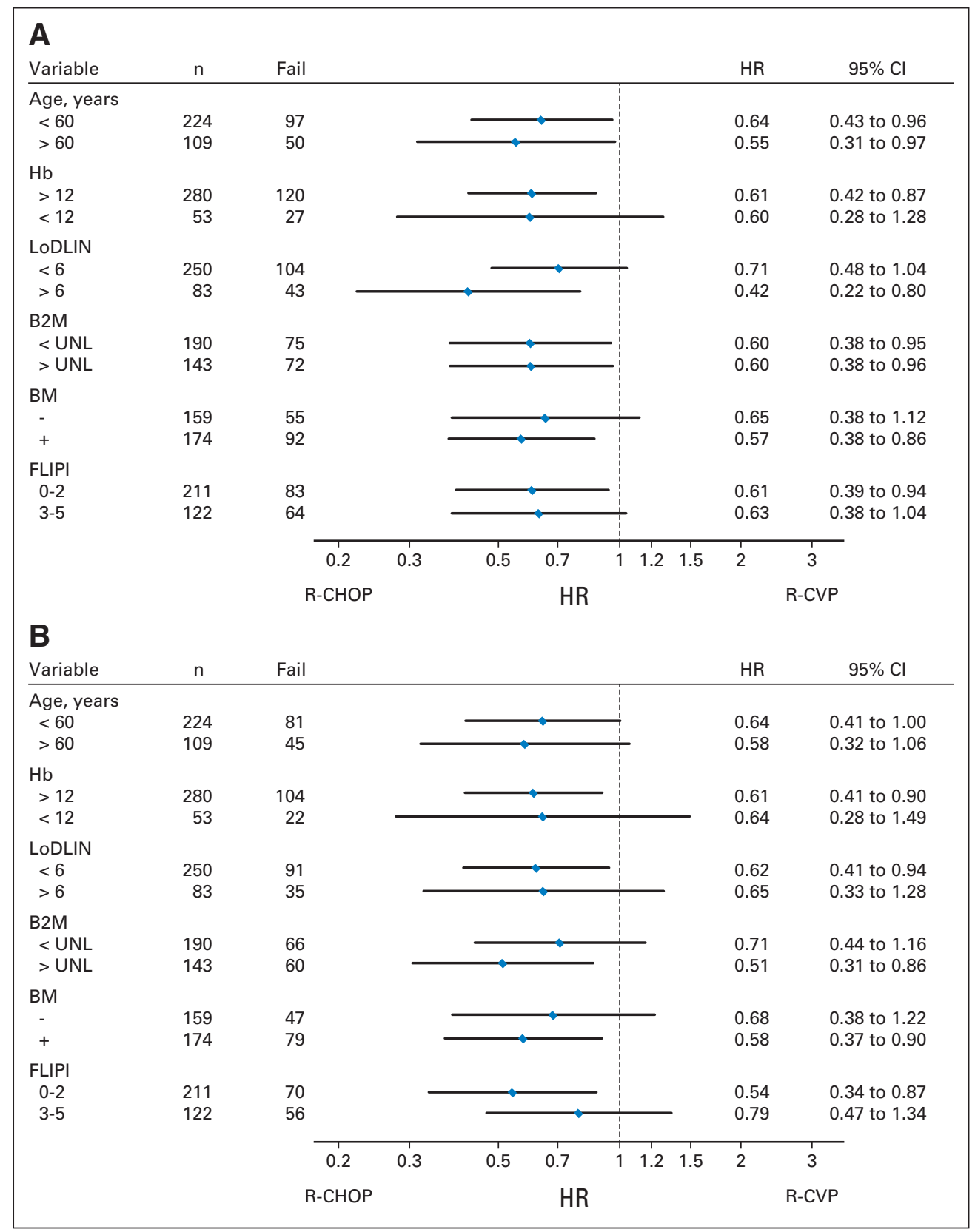

Fig 3. Post hoc exploratory analysis using Cox proportional hazards regression models of $(A)$ time to treatment failure and (B) progression-free survival among patients assigned to receive rituximab plus cyclophosphamide, doxorubicin, vincristine, and prednisone (R-CHOP) versus rituximab plus cyclophosphamide, vincristine, and prednisone (R-CVP). B2M, $\beta_{2}$-microglobulin; BM, bone marrow; FLIPI, Follicular Lymphoma International Prognostic Index; Hb, hemoglobin; $H R$, hazard ratio; LoDLIN, longest diameter of largest involved node. power caused by the low number of events $(\mathrm{n}=31)$ would have distorted any kind of result.

Recently, the PRIMA (Primary Rituximab and Maintenance) trial concluded that 2-year maintenance with $\mathrm{R}$ is to be considered standard therapy for patients responding to initial ICT. ${ }^{34}$ However, at the time of our study design, consensus regarding the role of maintenance had not been established. As a result, no maintenance was administered at end of therapy in our study.

Interestingly, 3-year PFS of the 458 responding patients in the FOLL05 study completely overlaps that of the 513 patients randomly assigned to the observation arm of the PRIMA study. PFS in the FOLL05 population validates the findings of the PRIMA study, ultimately confirming the benefit of 2 years of $\mathrm{R}$ maintenance after achievement of satisfactory response with ICT. Considering results from both the FOLL05 study and the PRIMA trial, we conclude that the current standard therapy for patients with advanced FL should be $\mathrm{R}-\mathrm{CHOP}$ followed by 2-year maintenance therapy with $\mathrm{R}$ for responding patients.

Recently bendamustine and lenalidomide were suggested as effective drugs for patients with FL. ${ }^{33,35,36}$ In particular, in a randomized trial conducted by STIL (Study Group Indolent Lymphomas), R-bendamustine (R-B) was compared with R-CHOP in a group of indolent and mantle-cell lymphomas. The study demonstrated that all patients, as well as the FL subgroup, treated with R-B had similar response rates compared with those achieved with R-CHOP, with improved PFS and toxicity profile. ${ }^{33}$ Thus, R-B may prove to be a valid alternative to $\mathrm{R}-\mathrm{CHOP}$ as a standard initial therapy for patients with FL. 
In conclusion, the FOLL05 trial supports R-CHOP as the reference regimen for initial management of patients with FL requiring active treatment. However, new questions have already arisen regarding both the efficacy of new promising drugs and best patient management at end of induction therapy. Available data suggest that quantitative assessment of response measured with either highly sensitive polymerase chain reaction-based arrays ${ }^{37}$ or, more recently, new functional imaging tools $\left[{ }^{18} \mathrm{~F}\right]$ fluorodeoxyglucose-positron emission tomography ${ }^{38-40}$ may be used to identify patients at different risks of progression, for whom different maintenance strategies are necessary.

\section{AUTHORS' DISCLOSURES OF POTENTIAL CONFLICTS OF INTEREST}

Although all authors completed the disclosure declaration, the following author(s) and/or an author's immediate family member(s) indicated a financial or other interest that is relevant to the subject matter under consideration in this article. Certain relationships marked with a " $U$ " are those for which no compensation was received; those relationships marked with a " $C$ " were compensated. For a detailed description of the disclosure categories, or for more information about ASCO's conflict of interest policy, please refer to the Author Disclosure Declaration and the Disclosures of Potential Conflicts of Interest section in Information for Contributors.
Employment or Leadership Position: None Consultant or Advisory Role: Umberto Vitolo, Roche (C) Stock Ownership: None Honoraria: None Research Funding: None Expert Testimony: None Other Remuneration: None

\section{AUTHOR CONTRIBUTIONS}

Conception and design: Massimo Federico, Stefano Luminari, Umberto Vitolo, Angelo Michele Carella, Alessandro Pulsoni, Stefano Sacchi, Maura Brugiatelli

Provision of study materials or patients: Massimo Federico, Stefano Luminari, Alessandra Tucci, Umberto Vitolo, Luigi Rigacci, Francesco Di Raimondo, Angelo Michele Carella, Alessandro Pulsoni, Francesco Merli, Luca Arcaini, Francesco Angrilli, Caterina Stelitano, Gianluca Gaidano, Matteo Dell'Olio, Vito Franco, Sara Galimberti, Stefano Sacchi Collection and assembly of data: Stefano Luminari, Alessandra Dondi, Alessandra Tucci, Umberto Vitolo, Luigi Rigacci, Francesco Di Raimondo, Angelo Michele Carella, Alessandro Pulsoni, Francesco Merli, Luca Arcaini, Francesco Angrilli, Caterina Stelitano, Gianluca Gaidano, Matteo Dell'Olio, Vito Franco, Sara Galimberti

Data analysis and interpretation: Massimo Federico, Stefano Luminari, Alessandra Dondi, Luigi Marcheselli, Stefano Sacchi

Manuscript writing: All authors

Final approval of manuscript: All authors

\section{REFERENCES}

1. Fisher RI, LeBlanc M, Press OW, et al: New treatment options have changed the survival of patients with follicular lymphoma. J Clin Oncol 23: 8447-8452, 2005

2. Marcus $R$, Imrie $K$, Belch $A$, et al: CVP chemotherapy plus rituximab compared with CVP as firstline treatment for advanced follicular lymphoma. Blood 105:1417-1423, 2005

3. Hiddemann W, Kneba M, Dreyling $M$, et al: Frontline therapy with rituximab added to the combination of cyclophosphamide, doxorubicin, vincris tine, and prednisone (CHOP) significantly improves the outcome for patients with advanced-stage follicular lymphoma compared with therapy with $\mathrm{CHOP}$ alone: Results of a prospective randomized study of the German Low-Grade Lymphoma Study Group. Blood 106:3725-3732, 2005

4. Herold M, Haas A, Srock S, et al: Rituximab added to first-line mitoxantrone, chlorambucil, and prednisolone chemotherapy followed by interferon maintenance prolongs survival in patients with advanced follicular lymphoma: An East German Study Group Hematology and Oncology Study. J Clin Oncol 25:1986-1992, 2007

5. Salles G, Mounier N, de Guibert S, et al: Rituximab combined with chemotherapy and interferon in follicular lymphoma patients: Results of the GELA-GOELAMS FL2000 study. Blood 112:48244831, 2008

6. Rigacci $L$, Federico $M$, Martelli $M$, et al: The role of anthracyclines in combination chemotherapy for the treatment of follicular lymphoma: Retrospective study of the Intergruppo Italiano Linfomi on 761 cases. Leuk Lymphoma 44:1911-1917, 2003

7. Kimby E, Björkholm M, Gahrton G, et al: Chlorambucil/prednisone vs. CHOP in symptomatic low-grade non-Hodgkin's lymphomas: A randomized trial from the Lymphoma Group of Central Sweden. Ann Oncol 5:67-71, 1994 (suppl 2)
8. Solal-Céligny $P$, Brice $P$, Brousse $N$, et al: Phase II trial of fludarabine monophosphate as firstline treatment in patients with advanced follicular lymphoma: A multicenter study by the Groupe d'Etude des Lymphomes de I'Adulte. J Clin Oncol 14:514-519, 1996

9. Coiffier B, Neidhardt-Bérard EM, Tilly $H$, et al: Fludarabine alone compared to CHVP plus interferon in elderly patients with follicular lymphoma and adverse prognostic parameters: A GELA studyGroupe d'Etudes des Lymphomes de I'Adulte. Ann Oncol 10:1191-1197, 1999

10. Hagenbeek A, Eghbali H, Monfardini S, et al: Phase III intergroup study of fludarabine phosphate compared with cyclophosphamide, vincristine, and prednisone chemotherapy in newly diagnosed patients with stage III and IV low-grade malignant non-Hodgkin's lymphoma. J Clin Oncol 24:15901596, 2006

11. Friedberg JW, Taylor MD, Cerhan JR, et al: Follicular Iymphoma in the United States: First report of the national LymphoCare study. J Clin Oncol 27:1202-1208, 2009

12. Federico $M$, Bellei $M$, Marcheselli $L$, et al: Follicular lymphoma international prognostic index 2: A new prognostic index for follicular lymphoma developed by the international follicular lymphoma prognostic factor project. J Clin Oncol 27:45554562, 2009

13. Harris NL, Jaffe ES, Diebold J, et al: The World Health Organization classification of neoplastic diseases of the haematopoietic and lymphoid tissues: Report of the Clinical Advisory Committee Meeting, Airlie House, Virginia, November 1997. Histopathology 36:69-86, 2000

14. Barosi G, Carella A, Lazzarino M, et al: Management of nodal indolent (non marginal-zone) nonHodgkin's lymphomas: Practice guidelines from the Italian Society of Hematology, Italian Society of Experimental Hematology and Italian Group for Bone Marrow Transplantation. Haematologica 90: 1236-1257, 2005
15. Solal-Céligny $P$, Roy $P$, Colombat $P$, et al: Follicular Lymphoma International Prognostic Index. Blood 104:1258-1265, 2004

16. Cheson BD, Horning SJ, Coiffier B, et al: Report of an international workshop to standardize response criteria for non-Hodgkin's lymphomas: $\mathrm{NCl}$-sponsored International Working Group. J Clin Oncol 17:1244-1253, 1999

17. Hryniuk WM: Average relative dose intensity and the impact on design of clinical trials. Semin Oncol 14:65-74, 1987

18. Sankoh AJ, Huque MF, Dubey SD: Some comments on frequently used multiple endpoint adjustment methods in clinical trials. Stat Med 16: 2529-2542, 1997

19. Altman DG, Schulz KF, Moher D, et al: The revised CONSORT statement for reporting randomized trials: Explanation and elaboration. Ann Intern Med 134:663-694, 2001

20. Kaplan E, Meier P: Nonparametric estimation from incomplete observations. J Am Stat Assoc 53:457-481, 1958

21. Cox D: Regression models and life tables. J R Stat Soc B 34:187-202, 1972

22. Schoenfeld D: Partial residuals for proportional hazard regression model. Biometrika 69:239241, 1982

23. Armitage $P$, Berry G: Statistical Methods in Medical Research (ed 2). Oxford, United Kingdom, Blackwell Scientific Publications, 1987

24. Cheson BD, Pfistner $B$, Juweid ME, et al: Revised response criteria for malignant lymphoma. J Clin Oncol 25:579-586, 2007

25. Marcus $R$, Imrie $K$, Solal-Celigny $P$, et al: Phase III study of R-CVP compared with cyclophosphamide, vincristine, and prednisone alone in patients with previously untreated advanced follicular Iymphoma. J Clin Oncol 26:4579-4586, 2008

26. Dana BW, Dahlberg $S$, Nathwani BN, et al: Long-term follow-up of patients with low-grade malignant lymphomas treated with doxorubicin-based 
chemotherapy or chemoimmunotherapy. J Clin Oncol 11:644-651, 1993

27. Baldini L, Brugiatelli $M$, Luminari $S$, et al: Treatment of indolent B-cell nonfollicular lymphomas: Final results of the LL01 randomized trial of the Gruppo Italiano per lo Studio dei Linfomi. J Clin Oncol 21:1459-1465, 2003

28. Nastoupil $L$, Sinha $R$, Byrtek $M$, et al: $A$ comparison of the effectiveness of first-line chemoimmunotherapy regimens for follicular lymphoma (FL) used in the United States. Blood 118, 2011 (abstr 97)

29. McLaughlin $P$, Rodriguez $M$, Hagemeister $F$, et al: Stage IV indolent lymphoma: A randomized trial of concurrent vs. sequential FND (fludarabine, mitoxantrone, dexamethasone) and rituximab, with interferon maintenance. Proc Am Soc Clin Oncol 22, 2003 (abstr 2269)

30. Zinzani PL, Pulsoni A, Perrotti A, et al: Fludarabine plus mitoxantrone with and without rituximab versus CHOP with and without rituximab as frontline treatment for patients with follicular lymphoma. J Clin Oncol 22:2654-2661, 2004

31. Vitolo $U$, Ladetto $M$, Boccomini $C$, et al: Brief chemoimmunotherapy R-FND with rituximab consolidation followed by randomization between rituximab maintenance vs. observation as first line treatment in elderly patients with advanced follicular lymphoma (FL): Final results of a prospective randomized trial by Italian Lymphoma Foundation (FIL). Blood 118, 2011 (abstr 777)

32. Sacchi S, Marcheselli L, Bari A, et al: Secondary malignancies after treatment for indolent nonHodgkin's lymphoma: A 16-year follow-up study. Haematologica 93:398-404, 2008

33. Rummel MJ, Niederle $N$, Maschmeyer G, et al: Bendamustine plus rituximab $(\mathrm{B}-\mathrm{R})$ versus $\mathrm{CHOP}$ plus rituximab (CHOP-R) as first-line treatment in patients with indolent and mantle cell lymphomas (MCL): Updated results from the StiL NHL1 study. J Clin Oncol 30:6s, 2012 (suppl; abstr 3)

34. Salles G, Seymour JF, Offner F, et al: Rituximab maintenance for 2 years in patients with high tumour burden follicular lymphoma responding to rituximab plus chemotherapy (PRIMA): A phase 3, randomised controlled trial. Lancet 377:42-51, 2011

35. Samaniego F, Hagemeister F, Mclaughlin P, et al: High response rates with lenalidomide plus rituximab for untreated indolent B-cell non-Hodgkin lymphoma, including those meeting GELF criteria. J Clin Oncol 29:511s, 2011 (suppl; abstr 8030)

36. Fowler $\mathrm{NH}, \mathrm{McLaughlin} \mathrm{P}, \mathrm{K}$ wak $\mathrm{L}$, et al: Lenalidomide and rituximab for untreated indolent
non-Hodgkin's lymphoma. J Clin Oncol 27:445s, 2009 (suppl; abstr 8548)

37. Ladetto $M$, De Marco F, Benedetti $F$, et al: Prospective, multicenter randomized GITMO/IIL trial comparing intensive (R-HDS) versus conventional (CHOP-R) chemoimmunotherapy in high-risk follicular lymphoma at diagnosis: The superior disease control of R-HDS does not translate into an overall survival advantage. Blood 111:4004-4013, 2008

38. Dupuis J, Meignan M, Julian A, et al: Significant prognostic impact of [18F]fluorodeoxyglucosePET scan performed during and at the end of treatment with R-CHOP in high-tumor mass follicular lymphoma patients: A GELA-GOELAMS study. Blood 118, 2011 (abstr 877)

39. Luminari S, Versari A, Quaresima $M$, et al: Role of FDG-PET as prognostic indicator in patients with follicular lymphoma (FL) after immunochemotherapy induction: A retrospective study from the Fondazione Italiana Linfomi. Blood 118, 2011 (abstr 2636)

40. Trotman J, Fournier M, Lamy T, et al: Positron emission tomography-computed tomography (PETCT) after induction therapy is highly predictive of patient outcome in follicular lymphoma: Analysis of PET-CT in a subset of PRIMA trial participants. J Clin Oncol 29:3194-3200, 2011

\section{Support}

Supported in part by grants from the Associazione Angela Serra per la Ricerca sul Cancro, Modena, Italy. 


\section{ERRATUM}

The April 20, 2013, article by Federico, et al entitled, "RCVP Versus R-CHOP Versus R-FM for the Initial Treatment of Patients With Advanced-Stage Follicular Lymphoma: Results of the FOLL05 Trial Conducted by the Fondazione Italiana Linfomi" (J Clin Oncol 31:1506-1513, 2013), contained errors.

In Table 1, a footnote gave the maximum dose of vincristine as "Maximum of $2 \mathrm{mg} / \mathrm{m}^{2}$," whereas it should have read, "Capped at a total dose of 2 mg."

Also, in the Patients and Methods section, under Statistics and Assessment of Efficacy, the second sentence of the third paragraph gave the final accrual as 531, whereas it should have been 534, as follows: "After enrollment of the first 214 patients, because actual accrual was faster than expected, the study was amended as follows: 4 years of uniform recruitment, 1 year of follow-up from last enrolled patient, and final accrual of $\mathbf{5 3 4}$ patients (178 patients per study arm)."

The online version has been corrected in departure from the print.

DOI: 10.1200/JCO.2014.55.9799; published April 1, 2014 